\title{
Amyloidosis Secondary to Rheumatoid Arthritis Associated with Plexiform Change in Bilateral Temporal Lobes
}

\author{
Yasunori Matsuki, Kimihiro Suzuki, Nobuhiko TanaKa, Tatsuo HiRose, Kouji HosoaI, \\ Makoto Kawakami, Toshiaki IshizuKa, Yasushi Kawaguchi, Toshihiko HidaKa and Haruo NaKamura
}

\begin{abstract}
A 70-year-old woman with rheumatoid arthritis (RA) and secondary amyloidosis presented repeated consciousness loss. The pathological findings at autopsy revealed multi-organic deposits of amyloid A-protein and so-called 'plexiform change' of blood vessels in bilateral temporal lobes. The arterial plexiform change, which is found in the lung specimen of primary pulmonary hypertension, might be a new pathological cerebrovascular change associated with RA.

(Internal Medicine 33: 764-767, 1994)
\end{abstract}

Key words: neurological symptom, cerebral infarction, cerebrovascular change

\section{Introduction}

Central nervous system involvement in rheumatoid arthritis (RA) is relatively uncommon. However, pathological investigations of case of RA associated with encephalopathy described cerebrovascular changes such as rheumatoid nodule formation, arteritis and amyloid deposition (1). Amyloidosis secondary to RA is resistant to treatment and the incidence of this complication has recently increased as a cause of death (2). Deposit of amyloid protein appeared in 20-30\% of autopsy cases with RA (3). This report describes a case of RA complicated with amyloidosis found to have a characteristic lesion; 'plexiform change' was found in the cerebral arteries at autopsy.

\section{Case Report}

A 70-year-old Japanese woman was diagnosed to have RA in 1972 at the age of 51. Oral corticosteroid and nonsteroidal anti-inflammatory drugs had been prescribed since then. She had a bilateral total hip replacement in 1986. In June 1991, she underwent replacement of the left femoral head for internal fracture of the femoral neck at the Department of Orthopedics of the National Defense Medical College Hospital. Appetite loss, diarrhea and dehydration appeared 1 month after the second operation. A pathological examination revealed deposits of amyloid A (AA)-protein in the rectal biopsy specimen. She was transferred to our department in late July 1991 . She had no serious illness except for RA and nothing contributory in the familial history. Upon transfer, physical examination of the patient yielded the following data: temperature, $37.4^{\circ} \mathrm{C}$; blood pressure, 132/72 $\mathrm{mmHg}$; pulse regular at 96 beats/minute. Purpura was found in the upper and lower bilateral extremities. The palpebral conjunctiva was remarkably anemic and bulbar conjunctiva revealed no icterus. Tongue was not enlarged. There was no lymphadenopathy and thyroid was not swollen. In thoracic auscultation, a grade II systolic heart murmur was audible. The liver edge was palpable for $3 \mathrm{~cm}$ in the midclavicular line, but the spleen was not palpable. Bilateral subluxations of wrist joints and deformities of fingers resulting from RA were noted, but there were no active arthritides. Neurological examination revealed no abnormalities except for mild dementia. Erythrocyte sedimentation rate was $75 \mathrm{~mm}$ in 1 hour. Urinalysis showed a $3+$ test for protein $(3.4 \mathrm{~g} /$ day in quantitative study). Occult blood was detected in feces. Laboratory examination yielded the following data: leukocyte count, $14,700 / \mathrm{mm}^{3}$; erythrocyte count, $2.52 \times 10^{4} / \mathrm{mm}^{3}$; hemoglobin, $5.5 \mathrm{~g} / \mathrm{dl}$; hematocrit, $24 \%$; platelet count, $54.7 \times 10^{4} / \mathrm{mm}^{3}$; serum creatinine, $1.1 \mathrm{mg} / \mathrm{dl}$; blood urea nitrogen, $26 \mathrm{mg} / \mathrm{dl}$; total protein, $5.3 \mathrm{~g} / \mathrm{dl}$; albumin, $2.9 \mathrm{~g} / \mathrm{dl}$; total cholesterol, $134 \mathrm{mg} / \mathrm{dl}$; amylase, $353 \mathrm{U} / \mathrm{l}$. Liver function tests and electrolytes were within normal limits. Results of immunological analyses were normal except for elevation of C-reactive protein to $1.0 \mathrm{mg} / \mathrm{dl}$. Prothrombin time (PT) and activated partial thromboplastin time (APTT) were prolonged to 17.7 seconds (control; 11.7) and 45.5 seconds (control; 34.4 ), respectively. The glomerular filtration rate (GFR) was decreased to the level of $17.8 \mathrm{ml} /$ minute. Chest roentogenogram showed cardiomegaly (cardiothoracic ratio: 68\%) and mild pulmonary congestion. An electrocardiogram (ECG) demonstrated transient atrial fi-

From the Internal Medicine I, National Defense Medical College, Tokorozawa Received for publication February 16, 1994; Accepted for publication July 29, 1994

Reprint requests should be addressed to Dr. Yasunori Matsuki, the Internal Medicine I, National Defense Medical College, 3-2 Namiki, Tokorozawa 359 

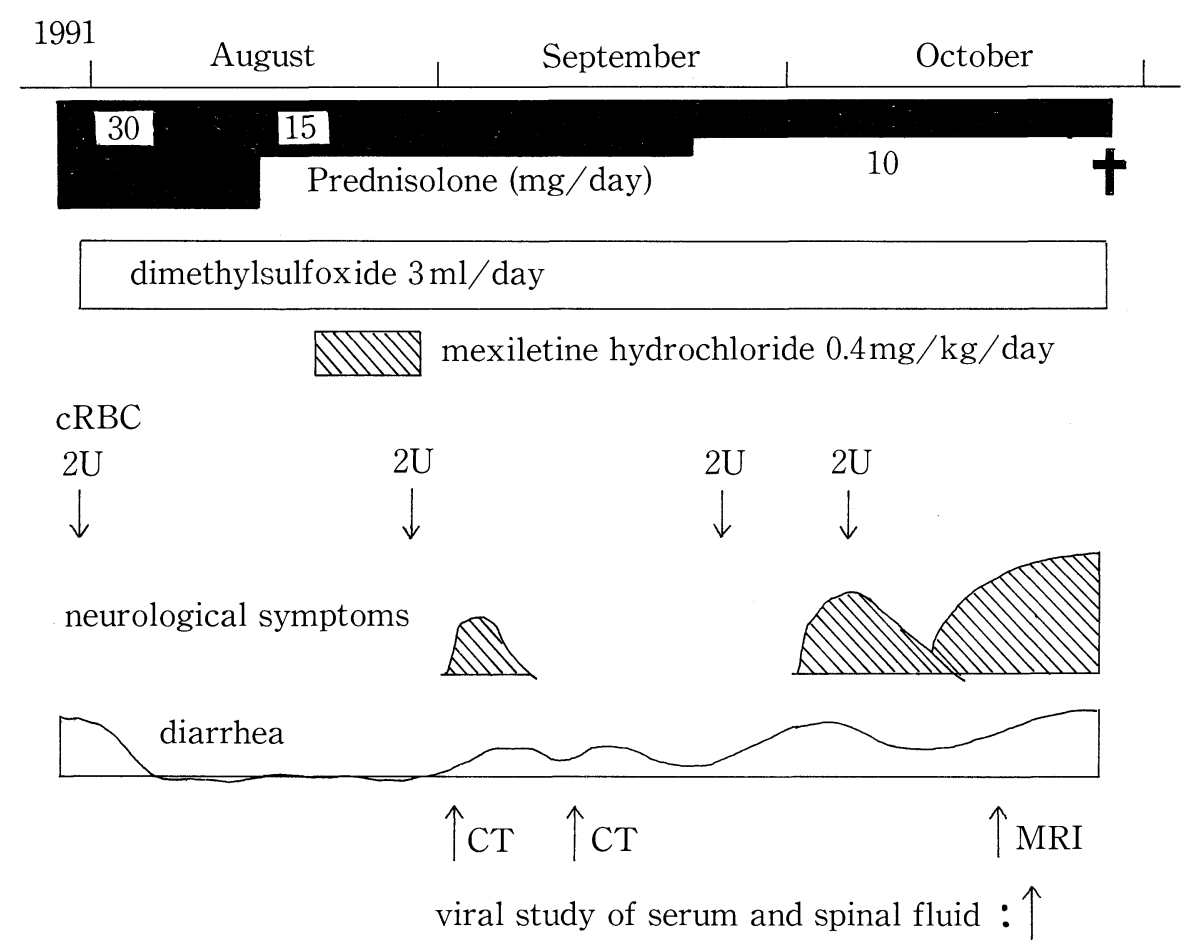

Fig. 1. Clinical course after transfer to the internal medicine ward. cRBC, concentrated red blood cell.

brillation and frequent ventricular extra systole. Asymmetrical septal hypertrophy was recognized by echocardiogram examination. Findings of ileus were not detected by plain abdominal X-ray films.

The patient received intravenous hyperalimentation due to severe dehydration from diarrhea and appetite loss. Treatments consisted of prednisolone; $30 \mathrm{mg} /$ day, dimethylsulfoxide (DMSO); $3 \mathrm{ml} /$ day, mexiletine hydrochloride; $0.4 \mathrm{mg} / \mathrm{kg} / \mathrm{day}$ and blood transfusion (Fig. 1). Diarrhea was improved 1 week after the start of these treatments. On the 38th day after transfer to our ward, the patient suddenly became unresponsive to verbal or pain stimulation and showed myoclonus of the gastrocnemius muscle and right upper conjugate deviation. At that time, the systemic blood pressure was 190/106 $\mathrm{mmHg}$ and ECG revealed atrial fibrillation with rapid ventricular response (heart rate; 150 beats/minute). The emergency computed tomography examination of the brain revealed atrophic change but there was no hemorrhage nor major infarction (Fig. 2). The consciousness loss was recovered within 5 days by conservative treatments, however the acute unconsciousness recurred 1 month later. On the 152 nd hospital day, magnetic resonance imaging (MRI) of the head was performed. $\mathrm{T}_{1}$-weighted images demonstrated low intensities in the bilateral temporal lobe cortexes and $\mathrm{T}_{2}$-weighted images depicted high intensities in the same areas. Viral study of the serum and cerebro-spinal fluid (CSF) revealed positive tests for IgG against herpes simplex 1 (1:640 and 1:2 in enzyme immunoassay, respectively). On CSF analysis, there was no increase in the cellular count or the protein level. She died suddenly on the 159th hospital day.

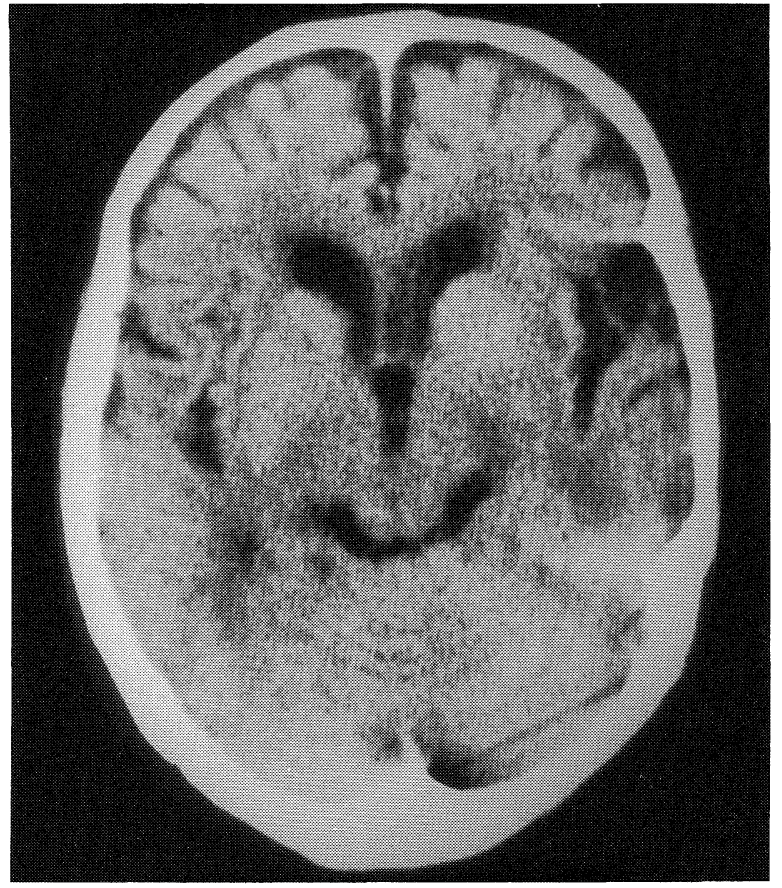

Fig. 2. Brain CT, after the first development of neurological symptoms (on the 38th hospital day), demonstrated cerebral atrophy.

Autopsy findings revealed deposition of amyloid protein in heart (including coronary arteries), kidney, liver, pancreas, spleen, bladder and gastrointestinal tract. The heart was en- 

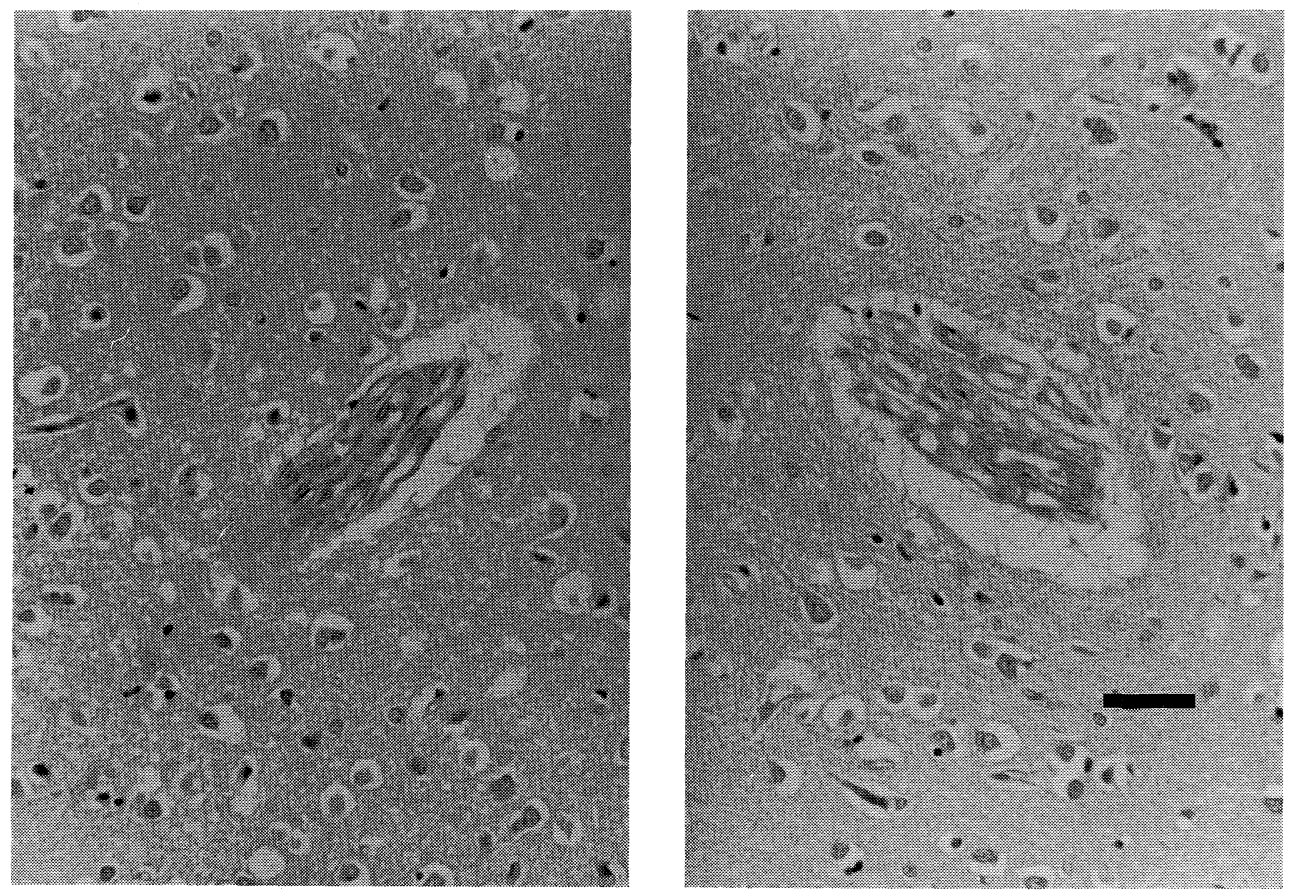

Fig. 3. Microscopic findings of the temporal lobes showed 'plexiform change' of the arterioles in the cerebral cortex $(\mathrm{HE}$ stain, $\times 200$, scale bar $=50 \mu \mathrm{m})$.

larged $(490 \mathrm{~g})$ and the ventricular wall was thickened $(2 \mathrm{~cm})$. Non-bacterial endocarditis, atherosclerotic changes of aorta, pulmonary edema, bone marrow hypoplasia, gastric ulcer, acute pancreatitis, spleen infarction and adrenal atrophy were also noted. In the brain, infarction was noted in bilateral temporal lobes. There was no obvious atherosclerotic change in the basilar artery. Upon microscopic examination, multiple 'plexiform change', of which the diameter ranged from $50 \mu \mathrm{m}$ to $125 \mu \mathrm{m}$, in small cerebral arteries was recognized in the infarction area (Fig. 3). In this areas of the temporal lobe, there were $5.1 \pm 0.8$ lesions per field at $100 \times$ magnification (mean \pm SD of 10 microscopic examinations). There was no inflammation such as encephalitis nor deposition of amyloid protein in the brain.

\section{Discussion}

The sudden onset of neurological symptoms in the present case might have been epileptic seizure and/or transient ischemia in the basilar artery. Electroencephalogram was not done. The patient was prone to transient cerebrovascular thrombosis due to atrial fibrillation. The anti-phospholipid syndrome, which is occasionally associated with RA (4), might have been responsible for the transient ischemia. Although no examinations for anticardiolipin antibody nor lupus anticoagulant were done, clotting abnormalities (PT and APTT elongation) were recognized in this case. Herpes simplex encephalitis might be also related to the neurological symptoms because of the elevation of antibody titer for herpes simplex in the serum and CSF.
However, there was no appropriate explanation for the transient clinical improvement without antiviral treatment and no definite findings of encephalitis in the brain specimen at autopsy.

The pathological findings of the cerebral arterioles in the present case were characteristic of plexiform lesion of the pulmonary artery found in primary pulmonary hypertension. If the lesions of the present case are classified according to the grading in Heath and Edwards pathological classification of pulmonary hypertension (5), the lesions would be grade IV. The formation occurs in conditions due to hypertension, hypoxia and chronic inflammation (6). In the present case, there was no past history of continuous systemic hypertension or hypoxia, but chronic inflammation due to RA might have bought about the cerebrovascular remodeling. Pulmonary plexiform lesions are formed by medial hypertrophy and intimal myofibroblast proliferation in muscular arteries. Electron microscopic examination of early pulmonary hypertension revealed that smooth muscle cells migrated from media into intima and became myofibroblasts (7). In such lesions in the lungs, narrowed vessel lumen is considered to be the cause of circulatory disturbance in the distributed areas (8). In the present case, the plexiform vascular change might have induced the surrounding infarctions in the temporal lobe.

On the contrary, plexiform change might account for the results of the ischemia. The localized hypoxia due to infarction (6) or the vascular regeneration after infarction might be responsible for such pathological change. In the present case, atrial fibrillation (or anti-phospholipid syndrome) might take part in the formation of temporal infarction. However, the 
plexiform vascular change associated with typical cerebral infarction based on arteriosclerosis is rarely reported.

In general, pathological cerebrovascular changes in RA are classified into two groups: (I) direct involvement by RA, and (II) secondary lesion due to vascular diseases and/or complications of RA (1). In direct arterial involvement in RA, there is rheumatoid nodule formation and arteritis, e.g., fibrinoid necrosis, perivascular inflammatory infiltration and onion skinlike formation (9). In the secondary lesions, there is infarction, hemorrhage and amyloid angiopathy. In the present case, there was neither rheumatoid nodule nor active arteritis at autopsy. However, inflammation might have been present in the early to the middle phase of her clinical course and might have caused the development of plexiform change. We speculate that this lesion might have caused the new type of cerebrovascular change classified in group I.

Amyloid deposition in the cerebrovascular wall might occur in amyloid angiopathy. Microscopic study of amyloid angiopathy reveals amyloid infiltration mainly in the media. In some cases, cluster formation of multiple arteriolar lumina (glomerular formations) is found that resembles the cerebrovascular change noted in this case (10). However, there was no amyloid protein deposition in the cerebral arteries of the present case. Therefore, the cause of the characteristic plexiform lesion in the cerebral arteries of this case should be irrelevant to amyloidosis.

Considering the autopsy findings, clinical manifestations, such as congestive heart failure, arrhythmia, gastrointestinal bleeding, diarrhea and proteinuria were the result of systemic amyloidosis. The heart contraction and conduction failure might possibly have been the cause of sudden death. The cardiac disturbance could be due to not only degeneration of myocardium but also to ischemic changes by amyloid deposition in the wall of coronary arteries (11).

In the treatment of amyloidosis, DMSO, alkylating agents (chlorambucil), colchicine and methotrexate associated with cyclophosphamide are used (12-14). Tatsuta et al described that colchicine inhibits AA protein synthesis and is considered to be effective for AA type amyloidosis, secondary to chronic inflammatory diseases (15). However, it is difficult to remove or decompose amyloid protein completely by any medication. In the present case, although diarrhea was ameliorated temporarily by administration of DMSO and corticosteroid, other clinical indicators such as the neurological symptoms, GFR, proteinuria and arrhythmia did not improve.

Acknowledgments: The authors appreciate Dr. Akira Senoo of the Department of Pathology II, National Defense Medical College, for his helpful suggestions regarding the pathological findings.

\section{References}

1) Kim RC, Collins GH. The neuropathology of rheumatoid disease. Hum Pathol 12: 5, 1981.

2) Husby G. Amyloidosis and rheumatoid arthritis. Clin Exp Rheumatol 3: 173, 1985.

3) Obana M. Clinical studies of amyloidosis complicated with rheumatoid arthritis with special reference to nephropathy. Jpn J Med 60: 274, 1990.

4) Lechner K. Lupus anticoagulants and thrombosis. in: International Society of Thrombosis and Haemostasis. Verstraete M, Vermylen J, Lijnen R, Aronout J, Eds. Leuven Univ Press, Leuven, 1987, p. 525.

5) Heath D, Edwards JE. The pathology of hypertensive pulmonary vascular diseases. Circulation 18: 533, 1958.

6) Tandon M, Warnock ML. Plexogenic angiopathy in pulmonary intralobar sequestrations: pathogenic mechanism. Hum Pathol 24: 263, 1993.

7) Smith P, Heath D, Yacoub M, Madden B, Caslin A, Gosney J. The ultrastructure of plexiogenic pulmonary arteriopathy. J Pathol 160: 111, 1990.

8) Harris P, Heath D. The Human Pulmonary Circulation. Third edition. Churchill Livingstone, New York, 1986, p. 254.

9) Ramos M, Mandybur TI. Cerebral vasculitis in rheumatoid arthritis. Arch Neurol 32: 271, 1975.

10) Mandybur TI. Cerebral amyloid angiopathy: The vascular pathology and complications. J Neuropathol Exp Neurol 45: 79, 1986.

11) Saltissi $S$, Kertes PJ, Julian DG. Primary cardiac amyloidosis in a young man presenting with angina pectoris. Br Heart J 52: 233, 1984.

12) Berglund $\mathrm{K}$, Keller C, Thysell H. Alkylating cytostatic treatment in renal amyloidosis secondary to rheumatic disease. Ann Rheum Dis 46: 757, 1987.

13) Gertz MA. Secondary amyloidosis (AA). J Intern Med 232: 517, 1992.

14) Scheinberg MA, Pernambuco JC, Benson MD. DMSO and colchicine therapy in amyloid disease. Ann Rheum Dis 43: 421, 1984.

15) Tatsuta E, Sipe JD, Shirahata T, Skinner M, Cohen AS. Colchicine inhibition of serum amyloid protein SAA and SAP synthesis in primary mouse liver cell culture. Arthritis Rheum 27: 349, 1984. 